LUNG CANCER

\title{
Real-time endobronchial ultrasound guided transbronchial needle aspiration for sampling mediastinal lymph nodes
}

\author{
F J F Herth, R Eberhardt, P Vilmann, M Krasnik, A Ernst
}

See end of article for authors' affiliations

Correspondence to: Professor F J F Herth Department of Internal Medicine, Pneumology and Critical Care

Medicine, Thoraxklinik, University of Heidelberg

Amalienstr 5, D-69126

Heidelberg, Germany; Felix.Herth@thoraxklinikheidelberg.de

Received 12 July 2005 Accepted 2 May 2006

Published Online First 31 May 2006

\begin{abstract}
Background: Transbronchial needle aspiration (TBNA) is an established method for sampling mediastinal lymph nodes to aid in diagnosing lymphadenopathy and in staging lung cancers. Real-time endobronchial ultrasound (EBUS) guidance is a new method of TBNA that may increase the ability to sample these nodes and hence to determine a diagnosis. A descriptive study was conducted to test this new method.

Methods: Consecutive patients referred for TBNA of mediastinal lymph nodes were included in the trial. When a node was detected, a puncture was performed under real-time ultrasound control. The primary end point was the number of successful biopsy specimens. Diagnostic results from the biopsies were compared with operative findings. Lymph node stations were classified according to the recently adopted American Thoracic Society scheme.

Results: From 502 patients (316 men) of mean age 59 years (range 24-82), 572 lymph nodes were punctured and $535(94 \%)$ resulted in a diagnosis. Biopsy specimens were taken from lymph nodes in region 2L (40 nodes), 2R (53 nodes), 3 (35 nodes), 4R ( 86 nodes), 4L (77 nodes), 7 (127 nodes), $10 R$ (38 nodes), 10L (43 nodes), $11 \mathrm{R}$ (40 nodes) and 11L (33 nodes). The mean (SD) diameter of the nodes was $1.6(0.36) \mathrm{cm}$ and the range was $0.8-3.2 \mathrm{~cm}$ (SD range $0.8-4.3$ ). Sensitivity was $94 \%$, specificity $100 \%$, and the positive predictive value was $100 \%$ calculated per patient. No complications occurred.

Conclusion: EBUS-TBNA is a promising new method for sampling mediastinal lymph nodes. It appears to permit more and smaller nodes to be sampled than conventional TBNA, and it is safe.
\end{abstract}

$\mathrm{N}$ on-small cell lung cancer (NSCLC) usually metastasises first to hilar and mediastinal lymph nodes. Subsequently, haematogenous metastasis to distant sites may occur. Because survival is inversely correlated with stage, a meticulous staging procedure is required to determine the treatment and prognosis. ${ }^{12}$

Mediastinal lymph node staging ${ }^{3}$ can be divided into imaging and sampling phases. Computed tomography, magnetic resonance imaging, and positron-emission tomography may be used to image mediastinal lymph nodes. ${ }^{4}$ Pathological sampling of suspicious lesions can be performed by mediastinoscopy, thoracoscopy, transthoracic needle aspiration, transbronchial needle aspiration (TBNA), and endoscopic ultrasonography with needle aspiration..$^{4-7}$

For many years mediastinoscopy has been regarded as the reference standard for sampling mediastinal lesions, but it is invasive, costly, and requires general anaesthesia. Although a standard cervical mediastinoscopy permits access to paratracheal lymph node stations (levels $2 \mathrm{R}, 2 \mathrm{~L}, 4 \mathrm{R}$, and $4 \mathrm{~L}$ ) and to the anterior subcarinal lymph node station (level 7), access to the posterior and inferior mediastinum is limited and requires either extended cervical mediastinoscopy or a thoracoscopy. ${ }^{8}$

To overcome the limitations of mediastinoscopy in accessing paratracheal and parabronchial lymph nodes (stations 2 , $3,4,7,10$ and 11 ), a new bronchoscope has been developed. This bronchoscope is equipped with a linear array transducer that allows real-time ultrasonic guidance of fine needle transbronchial biopsy. We used this new bronchoscope for 27 months during which we developed a method we call realtime endobronchial ultrasound guided transbronchial needle aspiration (EBUS-TBNA), and evaluated its clinical utility for visualising and sampling mediastinal and hilar lymph nodes under direct endobronchial ultrasound guidance. Here we describe our experience with this method.

\section{METHODS}

The study and prospective data collection was approved by the Institutional Review Boards at Thoraxklinik Heidelberg and Gentofte University Hospital, where all patients were examined by the authors. Written informed consent was obtained from all the patients in the study.

Between June 2002 and September 2004, patients with mediastinal or hilar lymphadenopathy referred for TBNA were enrolled in the study. The main indications for TBNA were the sampling and diagnosis of enlarged lymph nodes of unknown origin and lung cancer staging, especially the exclusion of N3 nodes. A chest radiograph and computed tomographic (CT) scan of the chest (plain and contrast enhanced) were routinely performed in all patients.

Conventional flexible bronchoscopy (model BF-7160 bronchoscope; Olympus, Tokyo, Japan) was first performed in a standard fashion to examine the tracheobronchial tree, followed by EBUS-TBNA using the new ultrasound biopsy bronchoscope (model XBF-UC260F-OL8; Olympus Ltd, Tokyo, Japan). Both bronchoscopy procedures were performed by the same operator.

All patients in whom a specific diagnosis was not determined from biopsy specimens obtained by EBUSTBNA underwent a surgical biopsy procedure (mediastinoscopy).

Abbreviations: EBUS, endobronchial ultrasound; NSCLC, non-small cell lung cancer; TBNA, transbronchial needle aspiration 


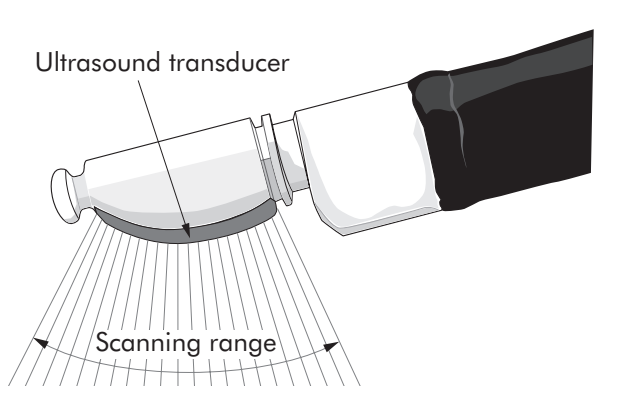

Figure 1 Scheme of the tip of the EBUS-TBNA bronchoscope.

\section{Prototype linear array ultrasonic bronchoscope}

Endobronchial ultrasound was performed using a prototype linear array ultrasonic bronchoscope. The instrument is similar to a standard bronchoscopic videoscope with an outer diameter of $6.9 \mathrm{~mm}$, a $2.0 \mathrm{~mm}$ instrument channel, and $30^{\circ}$ oblique forward viewing optics. An electronic convex array ultrasound transducer is mounted at the distal tip (fig l) and is covered by a water inflatable balloon sheath. Scanning is performed at a frequency of $7.5 \mathrm{MHz}$ and with a penetration of $50 \mathrm{~mm}$. The angle of view is $90^{\circ}$ and the direction of view is $30^{\circ}$ forward oblique. Image processing is performed by an Olympus ultrasound processor (EU-60).

A dedicated 22-gauge needle (XNA-202C; Olympus Ltd) was developed to perform transbronchial aspiration. The needle is also equipped with a stylet which is withdrawn after passing the bronchial wall, avoiding contamination during TBNA. The needle is mounted at the biopsy channel inlet of the endoscope before puncture. The needle exits the outer covering of the insertion tube at $20^{\circ}$. The needle can be visualised through the optics and on the ultrasound image.

\section{Biopsy procedure}

The probe was introduced through the mouth and vocal cords into the main carina. With the balloon partially inflated $(0.3-$ $0.5 \mathrm{ml}$ water), the regional lymph node stations of the middle mediastinum and hilar regions (stations 2, 3, 4, 7, 10, and $11)^{3}$ were systematically imaged and measured (short axis diameter) during slow withdrawal and rotation of the transducer.

Fine needle aspiration was performed by passing the dedicated prototype 22-gauge needle through the airway wall and into the lymph nodes under real-time ultrasound guidance. Needle punctures were performed using the "jabbing" method.5 Integrated power Doppler ultrasound was used to visualise and avoid potentially intervening vessels immediately before needle puncture (figs 2 and 3).

The aspirate was placed onto at least four glass slides, air dried, stained, and classified. Papanicolaou staining and light microscopy were performed by a cytopathologist who was blinded to the details of the patients. No rapid onsite cytology was performed. Two aspirates per node were obtained.

Diagnoses based on samples obtained through EBUSTBNA were confirmed by open thoracotomy, thoracoscopy, or clinical follow up. A positive cytological result of malignancy was accepted as evidence of cancer, and the patients were treated accordingly.

\section{Statistical methods}

The primary end point of the study was the percentage of biopsy specimens obtained that contained evaluable lymphocytes. The secondary end point was the percentage of confirmed diagnoses made possible with EBUS-TBNA. Diagnostic sensitivity, specificity, and accuracy were calculated using the standard definitions: the proportion of true

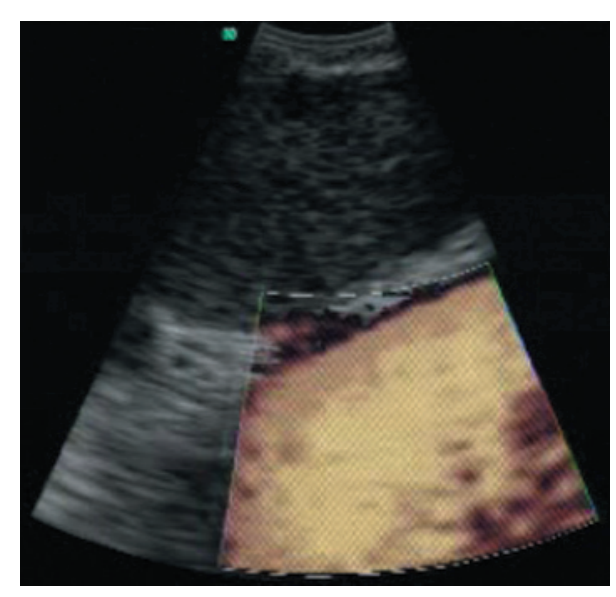

Figure 2 Colour Doppler image for detecting vessels before sampling the node.

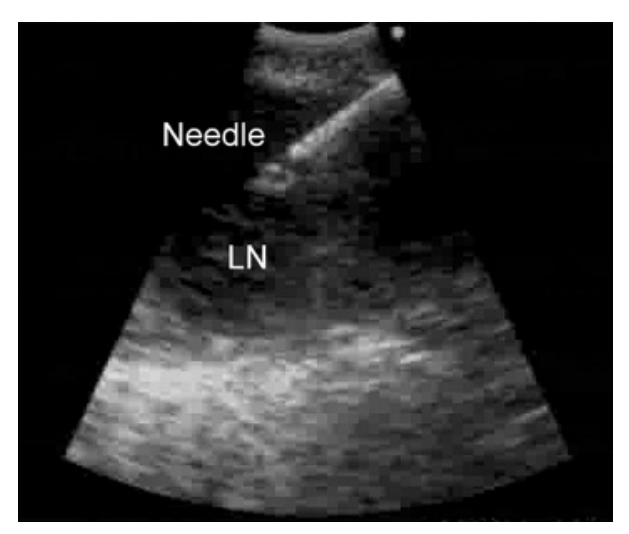

Figure 3 Endobronchial ultrasound image of the needle puncture.

positive results, true negative results, and all correct results, respectively. The unit of analysis was the patient.

\section{RESULTS}

Of the 502 patients who underwent TBNA, 186 were women and 316 were men (mean age 58.9 years, range 24-82). One hundred and eighty nine patients were examined under moderate sedation with midazolam (medium dose $3.5 \mathrm{mg}$ ) and topical anaesthesia ( $1 \%$ and 2\% lidocaine as needed), and 313 were examined under general anaesthesia and jet ventilation. The decision as to the type of anaesthesia used was at the discretion of the operator and depended mainly on which other procedures were planned for the same setting. There was no difference between institutions.

Among these patients, 572 lymph nodes were identified by CT scanning to be enlarged more than $1 \mathrm{~cm}$ and were punctured (mean 1.14/patient, range 1-3). Mean (SD) lymph node size was $1.6(0.36) \mathrm{cm}$ (range $0.8-3.2)$ as measured during the EBUS examination. The mean procedure time for EBUS-TBNA was 12.5 minutes (range 8-21). There was no difference in time required between types of anaesthesia.

Of the 572 nodes, 535 (93.5\%) were successfully biopsied and a specific diagnosis was established. The overall diagnostic yield was $93.5 \%$ (table 1). Six patients did have lymphocytes in the specimens but no specific diagnosis. Surgical biopsy in these cases confirmed sarcoidosis in two and malignancy in four. Table 2 lists the results of all biopsies.

Among the 37 nodes (6\%) in which the biopsy did not result in a diagnosis (including lymphocyte positive nodes), 
Table 1 Results of real-time EBUS-TBNA in 502 patients with mediastinal lymph nodes by location

\begin{tabular}{lcccc}
\hline Lymph node station & Nodes $(\mathbf{n})$ & Lymphocytes positive $(\mathbf{n})$ & $\begin{array}{l}\text { Diagnosis established from } \\
\text { biopsy }(\mathbf{n})\end{array}$ & Nodes diagnosed (\%) \\
\hline $2 R$ & 53 & 49 & 48 & 91 \\
$2 \mathrm{~L}$ & 40 & 36 & 36 & 90 \\
3 & 35 & 33 & 32 & 91 \\
$4 \mathrm{R}$ & 86 & 71 & 81 & 94 \\
$4 \mathrm{~L}$ & 77 & 123 & 74 & 96 \\
7 & 127 & 37 & 122 & 96 \\
IOR & 39 & 42 & 36 & 92 \\
$1 \mathrm{~L}$ & 43 & 36 & 42 & 97 \\
$11 \mathrm{R}$ & 40 & 29 & 36 & 90 \\
$11 \mathrm{~L}$ & 33 & 541 & 535 & 88 \\
Total & 572 & & 93.5 \\
\hline
\end{tabular}

35 were found on surgery to have a malignancy and two had sarcoidosis.

The results did not differ when controlled for the type of anaesthesia used. In patients who received a general anaesthetic the accuracy was $94.8 \%$, positive predictive value (PPV) $100 \%$, negative predictive value (NPV) $11 \%$, sensitivity $95 \%$, and specificity $100 \%$. In patients who received moderate sedation the accuracy was $93.6 \%$, PPV 100\%, NPV $14 \%$, sensitivity $93.5 \%$, and specificity $100 \%$.

Analysing the diagnostic yield for the 502 patients rather than the nodes, we were able to establish a definitive diagnosis in 470 patients (93\%). The sensitivity, specificity, and accuracy were 94\%, 100\%, and 94\%, respectively; PPV was $100 \%$ and NPV $11 \%$.

No complications were associated with EBUS-TBNA.

\section{DISCUSSION}

Chest physicians often need to assess enlarged mediastinal lymph nodes. ${ }^{12}$ Lymph nodes may be enlarged for a variety of inflammatory, infectious, or malignant reasons, and it is important to ascertain a diagnosis or-in the case of malignancy-to determine the stage of the disease before making treatment decisions. ${ }^{9}$

Mediastinoscopy has long been the mainstay of mediastinal lymph node sampling but it has several disadvantages. As a surgical procedure it requires general anaesthesia and the use of an operating room. It is also difficult to perform a second time in a given patient. The reach of the procedure is limited as lymph nodes in the posterior carina and hilar stations are generally inaccessible. Furthermore, although it is currently the reference standard, the specificity and sensitivity of mediastinoscopy are not optimal. ${ }^{8-10}$

Interest in non-surgical staging has been increasing since endoscopic needle aspiration of mediastinal lymph nodes has been possible. ${ }^{11-13}$ TBNA is an established bronchoscopic

Table 2 Diagnosis obtained by surgery and by EBUSTBNA study patients $(n=502)$; a definitive diagnosis was made by endoscopic means in 470

\begin{tabular}{lll}
\hline & Overall & $\begin{array}{l}\text { Definitive diagnosis } \\
\text { by EBUS } \\
\text { N (\%) }\end{array}$ \\
& N & \multicolumn{1}{c}{$(138(93 \%)$} \\
\hline Squamous cell carcinoma & 148 & $150(96 \%)$ \\
Adenocarcinoma & 156 & $49(88 \%)$ \\
Large cell carcinoma & 56 & $9(90 \%)$ \\
NSCLC & 10 & $119(96 \%)$ \\
SCLC & 123 & $2(33 \%)$ \\
Sarcoidosis & 6 & $3(100 \%)$ \\
Tuberculosis & 3 & $470(94 \%)$ \\
Total & 502 & \\
\hline
\end{tabular}

technique but it remains underused and the biopsy yield varies widely, perhaps because of the long learning curve and its associated frustrations. Additionally, conventional TBNA is a fairly blind technique that does not allow the target to be visualised, making smaller lymph nodes and nodes in some locations more difficult to access.

The introduction of endo-oesophageal ultrasound guided and endobronchial ultrasound guided needle aspiration and the resulting increase in yield has further enhanced this diagnostic instrument. ${ }^{714-19}$

Endoscopic imaging with simultaneous ultrasound scanning has several advantages over mediastinoscopy: there is no need for surgery or general anaesthesia; it can be performed repetitively in the same person; and, depending on which endoscopic modality is used, lymph node stations not surgically accessible are within reach. ${ }^{15} 182021$ Before the development of real-time EBUS-TBNA bronchoscopy, TBNA was limited to "blind" needle puncture guided by static CT scans. This method is highly operator dependent and the successful sampling rate varies between $20 \%$ and $80 \%{ }^{7-9}$ We have recently shown that a mechanical radial ultrasound mini-probe, introduced through the working channel of a flexible bronchoscope, can be used to localise target lymph nodes before sequential blind biopsy. ${ }^{17}{ }^{18}$ This method increased the sampling success rate to $86 \% .{ }^{11}$ Although this technique has improved TBNA yields, it does not allow realtime ultrasound guided control of needle puncture, and inadvertent puncture of vascular structures is a risk.

In this study we have shown that real-time EBUS guided fine needle aspiration can be used to identify and aspirate lymph nodes adjacent to the trachea and main bronchi from lymph node stations $2,3,4,7,10$, and 11 with a sampling success rate of more than $90 \%$. Our findings also support the recommendations that negative TBNA needs to be followed up with a more definitive diagnostic procedure (as evidenced by the low NPV).

We did note some drawbacks with the bronchoscope. The direction of view of the scope is $30^{\circ}$ forward oblique which makes manipulation difficult. From our experience, this new bronchoscope requires at least 10 manipulations for an experienced bronchoscopist to be able to insert it smoothly and to obtain clear images. Subaortic and paraoesophageal lymph nodes (levels 5, 6, 8, 9) were not accessible with EBUS-TBNA. However, most mediastinal and hilar lymph nodes occur along the airway so they should be accessible using EBUS-TBNA. For lymph nodes not accessible with EBUS-TBNA, endoscopic guided fine needle aspiration may need to be performed. ${ }^{74-16}$

Three papers have so far been published on this procedure. Krasnik et $a l^{22}$ reported on 11 patients in whom 15 lesions were punctured without complications. The lesions were located as follows: four in region $10 \mathrm{~L}$, four in region $10 \mathrm{R}$, one 
in region $4 \mathrm{~L}$, three in region $4 \mathrm{R}$, one in region 1 , one in region 7 , and one in region $2 \mathrm{R}$. The lesions ranged from $7 \mathrm{~mm}$ to $80 \mathrm{~mm}$. Biopsies obtained by EBUS-FNA showed malignant cells in 13 lesions and benign cells in two. Yasufuku et al ${ }^{23}$ examined 70 patients with 58 mediastinal lymph nodes and 12 hilar lymph nodes. The sensitivity, specificity, and accuracy of EBUS-TBNA in distinguishing benign from malignant lymph nodes were $95.7 \%, 100 \%$, and $97.1 \%$, respectively. There were no complications. In a recent paper by Rintoul et al, ${ }^{24}$ EBUS-TBNA was used in 18 patients. Cytological examination revealed node $(\mathrm{N}) 2 / \mathrm{N} 3$ disease in 11 patients and provided a primary diagnosis in eight. The cytology results for EBUS-TBNA samples were negative in six patients and mediastinoscopy or clinical follow up confirmed this result in four. The sensitivity, specificity, and accuracy of EBUS-TBNA were $85 \%, 100 \%$, and $89 \%$, respectively.

Our study establishes the method of performing real-time EBUS-TBNA in a large group of patients and has confirmed our assumption that the use of EBUS for TBNA results in a high success rate for accessing lymph nodes and a high diagnostic yield. It allowed for reliable biopsy sampling of small nodes and of nodes in difficult locations. When using EBUS, lymph node location and size did not influence the success of actually "hitting" the intended node. This is in contrast to conventional TBNA where there is a significant difference in diagnostic success depending on node location. This gives additional support to the assumption that imaging guidance is beneficial for TBNA.

In conclusion, we believe that TBNA of mediastinal and hilar lymph nodes under direct real-time EBUS guidance using the new ultrasound bronchoscope is a safe and effective means of obtaining biopsy specimens from these nodes.

\section{Authors' affiliations}

F J F Herth, R Eberhardt, A Ernst, Department of Pneumology and Critical Care Medicine, Thoraxklinik, University of Heidelberg, Germany P Vilmann, Department of Surgical Gastroenterology, Gentofte University Hospital, Copenhagen, Denmark

M Krasnik, Department of Cardiothoracic Surgery, Gentofte University Hospital, Copenhagen, Denmark

A Ernst, Interventional Pulmonology, Beth Israel Deaconess Medical Center, Harvard Medical School, Boston, USA

The prototype endobronchial ultrasound probe was loaned to the authors by Olympus Ltd, Tokyo, Japan for the duration of the study. None of the authors has any financial stake in Olympus Ltd, Tokyo, Japan.

\section{REFERENCES}

1 Sihoe AD, Yim AP. Lung cancer staging. J Surg Res 2004; 117:92-106.

2 Spira A, Ettinger DS. Multidisciplinary management of lung cancer. N Engl'J Med 2004;350:379-92.

3 Mountain CF, Dresler CM. Regional lymph node classification for lung cancer staging. Chest 1997;111:1718-23.

4 Toloza EM, Harpole L, Detterbeck F, et al. Invasive staging of non-small cell lung cancer: a review of the current evidence. Chest 2003;123:157-66.

5 Garpestad E, Goldberg S, Herth F, et al. CT fluoroscopy guidance for transbronchial needle aspiration: an experience in 35 patients. Chest 2001;119:329-32.

6 Mehta AC, Kavuru MS, Meeker DP, et al. Transbronchial needle aspiration for histology specimens. Chest 1989;96:1268-72.

7 Vilmann P, Hancke S. A new biopsy handle instrument for endoscopic ultrasound guided biopsy. Gastrointest Endosc 1996;43:238-42.

8 Hoffmann H. Invasive staging of lung cancer by mediastinoscopy and videoassisted thoracoscopy. Lung Cancer 2001;34:3-5.

9 Luke WP, Pearson FG, Todd TR, et al. Prospective evaluation of mediastinoscopy for assessment of carcinoma of the lung. J Thorac Cardiovasc Surg 1986;91:53-6.

10 Coughlin M, Deslauriers J, Beaulieu M, et al. Role of mediastinoscopy in pretreatment staging of patients with primary lung cancer. Ann Thorac Surg 1985:40:556-60.

11 Gasparini S, Zuccatosta L, DeNictolis M. Transbronchial needle aspiration of mediastinal lesions. Monaldi Arch Chest Dis 2000;55:29-32.

12 Mehta AC, Meeker DP. Transbronchial needle aspiration for histology specimens. In: Wang KP, Mehta AC, eds. Flexible bronchoscopy. Cambridge: Blackwell Science, 1995: 199-205.

13 Wagner ED, Ramzy I, Greenburg SO, et al. Transbronchial fine needle aspiration: reliability and limitations. Am J Clin Pathol 1989:92:36-41.

14 Wiersema MJ, Vilmann P, Giovannini M, et al. Endosonography guided fine needle aspiration biopsy: Diagnostic accuracy and complication assessment. Gastroenterology 1997; 112:1087-95.

15 Pedersen BH, Vilmann P, Milman N, et al. Endoscopic ultrasonography with guided fine needle aspiration biopsy of a mediastinal mass lesion. Acta Radiol 1995;36:326-8.

16 Silvestri GA, Hoffman BJ, Bhutani MS, et al. Endoscopic ultrasound with fineneedle aspiration in the diagnosis and staging of lung cancer. Ann Thorac Surg 1996;61:1441-6.

17 Herth F, Becker HD, Ernst A. Conventional vs endobronchial ultrasoundguided transbronchial needle aspiration: a randomized trial. Chest 2004; 125:322-5.

18 Herth FJ, Becker HD, Ernst A. Ultrasound-guided transbronchial needle aspiration: an experience in 242 patients. Chest 2003;123:604-7.

19 Okamoto H, Watanabe K, Nagatomo A, et al. Endobronchial ultrasonography for mediastinal and hilar lymph node metastases of lung cancer. Chest 2002;121:1498-506.

20 Herth F, Becker HD. Endobronchial ultrasound of the airways and the mediastinum. Monaldi Arch Chest Dis 2000;55:36-45.

21 Falcone F, Fois F, Grosso D. Endobronchial ultrasound. Respiration 2003;70:179-94.

22 Krasnik M, Vilmann P, Larsen SS, et al. Preliminary experience with a new method of endoscopic transbronchial real time ultrasound guided biopsy for diagnosis of mediastinal and hilar lesions. Thorax 2003;58:1083-8.

23 Yasufuku K, Chiyo M, Sekine Y, et al. Real-time endobronchial ultrasoundguided transbronchial needle aspiration of mediastinal and hilar lymph nodes. Chest 2004;126:122-8.

24 Rintoul RC, Skwarski KM, Murchison JT, et al. Endobronchial and endoscopic ultrasound-guided real-time fine-needle aspiration for mediastinal staging. Eur Respir J 2005;25:416-21. 